\title{
3-D Air Flow in a Toroidal Natural Convection Loop
}

\author{
Manal H. Saleh, PhD \\ Faculty in Engineering College \\ University of Baghdad \\ Mechanical Department
}

\begin{abstract}
Heat transfer characteristics of air flows in concentric toroidal annular tubes filled with porous media were numerically investigated. The toroidal annulus is constant walls temperature where the outer wall temperature is lower than the inlet temperature. The numerical methodology was based on the finite difference approximation method. The computations were performed for vertical and horizontal toroidal ring, four toroidal length and four radius ratios cases for a modified Rayleigh number range $(10 \leq \mathrm{Ra} \leq 5000)$. The inner tube radius ranged from 0.15 to $0.4 \mathrm{~m}$ with the radius ratios $\operatorname{Rr} 0.333,0.555,0.777$ and 0.888 and a length of $0.05,0.1,0.15$ and $0.2 \mathrm{~m}$. The local Nusselt number in the angular direction for both walls and the average Nusselt number in concentric toroidal annular tubes were obtained. In addition the isothermal lines and the streamlines were investigated. The results show that The vertical toroidal loop is more suitable than the horizontal one because the heat transfer is less so the temperature values will be higher and remain high for a longer time which is required in such devices The best design of such devices is to take $\mathrm{L}=0.05$ with inner radius of $0.15 \mathrm{~m}$ and to use it at $\delta=0$ (vertical)
\end{abstract}

\section{General Terms}

Cp: Specific heat at constant pressure $\left(\mathrm{kJ} / \mathrm{kg}^{\circ} \mathrm{C}\right)$, g: Acceleration due to gravity $\left(\mathrm{m} / \mathrm{s}^{2}\right), \mathrm{k}_{\mathrm{f}}$ : Thermal conductivity of the fluid $(\mathrm{W} / \mathrm{m} \mathrm{K})$, ks: Thermal conductivity of the solid $(\mathrm{W} / \mathrm{m} \mathrm{K}), \mathrm{k}_{\mathrm{eff}}$ : Effective thermal conductivity of the porous media $(\mathrm{W} / \mathrm{m} \mathrm{K}), \mathrm{K}$ : Permeability $\left(\mathrm{m}^{2}\right)$, 1: toroidal gap length $(\mathrm{m}), \mathrm{L}$ : Dimensionless toroidal gap length, $\mathrm{Nu}_{\text {Local } 1}$ : Local Nusselt number on the inner surface, $\mathrm{Nu}_{\text {Local } 2}$ : Local Nusselt number on the outer surface, $\mathrm{Nu}_{1}$ : Mean Nusselt number on the inner surface, $\mathrm{Nu}_{2}$ : Mean Nusselt number on the outer surface, p: Pressure (N/m2), q: Local heat flux (m), r: Radial coordinate $(\mathrm{m}), \mathrm{R}$ : Dimensionless radial coordinate, Ra*: Modified Rayleigh number, Rr: Radius ratio, T: Temperature $(\mathrm{K})$, ur,u $\phi$, uz: velocity component in $\mathrm{r}, \phi$ and $\mathrm{z}$ - direction $(\mathrm{m} / \mathrm{s})$, Ur, U $\phi, U z$ : Dimensionless velocity component in R, $\phi$ and $\mathrm{Z}$ direction, $\mathrm{x}, \mathrm{y}, \mathrm{z}$ : Cartesian coordinate system $(\mathrm{m}), \mathrm{Z}$ : Dimensionless axial coordinate, $\alpha_{\mathrm{f}}$ : Fluid thermal diffusivity $\left(\mathrm{m}^{2} / \mathrm{s}\right), \alpha_{\mathrm{s}}$ : Solid thermal diffusivity, $\alpha_{\text {eff. }}$ : Effective thermal diffusivity $\left(\mathrm{m}^{2} / \mathrm{s}\right), \beta$ : Volumetric thermal expansion coefficient $(1 / \mathrm{K}), \theta$ : Dimensionless temperature, $\psi \mathrm{r}, \psi \phi, \psi \mathrm{z}$ : Vector potential component in R, $\phi$ and $\mathrm{Z}$ - direction, $\mu_{f}$ : Dynamic viscosity of fluid (Pa.s), FAI: angular direction.

\section{Keywords}

Toroidal annular, Porous media, Natural Convection.

\section{INTRODUCTION}

Many researches on natural convection in conventional configurations of square, rectangular and another configurations enclosure are done, as evidenced by [1-4].
[5] Investigated numerically the transient laminar natural convection regimes occurring in a thermal convection loop heated from below and cooled from above for a wide range of Rayleigh numbers. The upper half of the loop is cooled and maintained at a constant low temperature while the lower half of the loop is heated and maintained at a constant high temperature. The finite volume method is used to solve the system of governing flow equations.

[6] Described a new working principle for a radio frequency (RF) hyperthermia applicator, suitable for heating large cylindrical volumes of coaxial loads. The system includes a toroidal inductor as an active device producing an almost uniform $\mathrm{E}$ field, which is polarized along the toroidal $Z$ axis in the space inside the toroidal ring where the conductive tissue is placed. This E field produces axial RF currents which, in turn, produce the required heating, provided that a closed loop is formed by electrically connecting the conductive load through capacitive ring (CR) electrodes to form an electric circuit closed externally to the toroidal inductor.

[7] Study experimentally the free and mixed convection heat transfer from inductor toroids to determine the averaged Nusselt number. The experimental program range was conducted in the Reynolds number range $4000 \leq R e \leq$ 13000 and $3 \times 10^{6} \leq R a \leq 7 \times 10^{6}$. General models were proposed for natural and mixed convection from the inductor toroids in different orientations.

\section{OBJECTIVE OF THE RESEARCH}

In this research an investigation are made for the natural convection heat transfer in a toroidal ring filled with porous media (silica sand) which is used as a medical device used for remedy. In this device a heater is used to heat the inner ring and the heat will be transferred to the sand which detains the heat for many hours. The computations were performed for four toroidal length and four radius ratios cases. The inner toroidal ring radius ranged from 0.15 to $0.4 \mathrm{~m}$ with the radius ratios $\operatorname{Rr} 0.333,0.555,0.777$ and 0.888 and a length of 0.05 , $0.1,0.15$ and $0.2 \mathrm{~m}$ for a modified Rayleigh number range $(10 \leq \mathrm{Ra} \leq 5000)$.

\section{MATHEMATICAL MODEL}

The device considered is a toroidal (ring) loop. The gap of the toroidal loop is filled with porous media.

The isometric of the toroidal loop is illustrated in the Fig.1. In order to model the incompressible flow in the porous medium, the steady-state equations of the Darcy flow model, namely, mass, momentum (Darcy) and energy conservation laws and the Boussinesq's approximation were employed. These equations in vectorial notation were given by [8] 


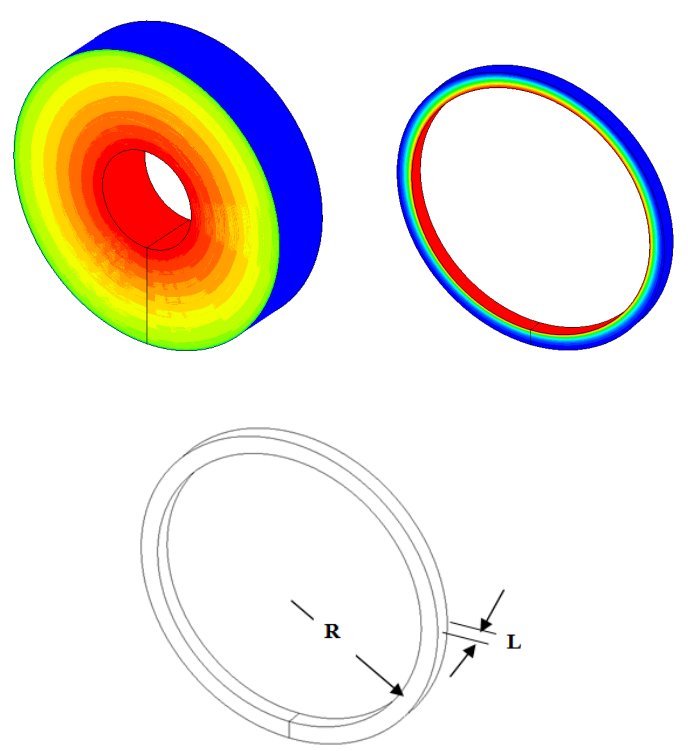

Fig. 1 The isometric and the coordinate sketch of the toroidal loop for different lengths and radius ratios

\section{GOVERNING EQUATIONS}

The conservation equations of mass, momentum and energy in steady state and the supplementary equation are:

$\rho=\rho_{2}\left\{1-\beta\left(T-T_{2}\right)\right\}$

Where:

$\beta=\frac{1}{\rho} \frac{\partial \rho}{\partial T}$

$\beta$ Is the thermal coefficient of the volume expansion, this constant is evaluated at $T_{2}$ which is the temperature at the inner surface of the toroidal loop, $\rho_{2}$ is the density at $T_{2}$ and $\rho$ is the density at T, [9]. This technique is called Boussinesq's approximation.

\subsection{Mass Conservation}

$\frac{\partial u_{r}}{\partial r}+\frac{u_{r}}{r}+\frac{1}{r} \frac{\partial u_{\emptyset}}{\partial \emptyset}+\frac{\partial u_{z}}{\partial z}$

\subsection{Momentum Equations}

Darcy flow model in three dimensions is used in the porous media as in [10] which states that the volume average velocity through the porous material is proportional with the pressure gradient.

\subsubsection{Momentum Equation in Radial Direction $u_{r}=\frac{K}{\mu_{f}}\left[-\frac{\partial p}{\partial r}-\rho g \cos \emptyset \cos \delta\right]$}

\subsubsection{Momentum Equation in Angular Direction \\ $u_{\emptyset}=\frac{K}{\mu_{f}}\left[-\frac{\partial p}{\partial \emptyset}+\rho g \sin \emptyset \cos \delta\right]$}

\subsubsection{Momentum Equation in Axial Direction} $u_{z}=\frac{K}{\mu_{f}}\left[-\frac{\partial p}{\partial z}-\rho g \sin \delta\right]$

\subsection{Energy Equation}

$\frac{\partial\left(\rho C_{p} T\right)}{\partial t}+u_{r} \frac{\partial\left(\rho C_{p} T\right)}{\partial r}+\frac{u_{\emptyset}}{r} \frac{\partial\left(\rho C_{p} T\right)}{\partial \emptyset}$

$+u_{z} \frac{\partial\left(\rho C_{p} T\right)}{\partial z}=\frac{1}{r} \frac{\partial}{\partial r}\left\{r \frac{\partial(k T)}{\partial r}\right\}+\frac{1}{r^{2}} \frac{\partial^{2}(k T)}{\partial \emptyset^{2}}$

$+\frac{\partial^{2}(k T)}{\partial z^{2}}+\mu \Phi$

Where $\Phi$ is viscous dissipation function.

A vorticity vector $\Omega$ and a vector potential $\Psi$ with its components are [11]:

$\Psi=\left(\psi_{r}, \psi_{\phi}, \psi_{z}\right)$

Defined by:

$U=\alpha_{e f f} . \nabla \mathrm{X} \Psi$

$\nabla^{2} \psi_{r}=\frac{1}{R} \frac{\partial U_{Z}}{\partial \emptyset}-\frac{\partial U_{\emptyset}}{\partial Z}$

$\nabla^{2} \psi_{\varnothing}=\frac{\partial U_{r}}{\partial Z}-\frac{\partial U_{z}}{\partial R}$

$\nabla^{2} \psi_{z}=\frac{1}{R} \frac{\partial\left(R U_{\emptyset}\right)}{\partial R}-\frac{1}{R} \frac{\partial U_{r}}{\partial \emptyset}$

\section{NON DIMENSIONAL VARIABLES}

Convert the governing equations to the dimensionless form using $r_{2}$ as a characteristic length [12]. The dimensionless magnitudes defined as follow:

$R=\frac{r}{r_{2}}, Z=\frac{Z}{r_{2}}, U_{r}=\frac{u_{r} l}{\alpha_{e f f .}}, U_{\emptyset}=\frac{u_{\emptyset} l}{\alpha_{e f f .}}, U_{z}=\frac{u_{z} l}{\alpha_{e f f} .}$

$\theta=\frac{\left(T-T_{2}\right)}{\left(T_{1}-T_{2}\right)}, P=\frac{p K l}{\alpha_{e f f .} \mu_{f} r_{2}}, R_{a}^{*}=\frac{g \beta K\left(T_{1}-T_{2}\right)\left(r_{2}-r_{1}\right)}{\alpha_{e f f .} \vartheta}$

Substitute these dimensionless magnitudes in the governing equations. Alternative expressions of equation (3) may be written in terms of $\psi_{r}, \psi_{\emptyset}, \psi_{z}$ as:

$U_{r}=\left(\frac{1}{R} \frac{\partial \psi_{z}}{\partial \emptyset}-\frac{\partial \psi_{\emptyset}}{\partial Z}\right)$

$U_{\emptyset}=\left(\frac{\partial \psi_{r}}{\partial Z}-\frac{\partial \psi_{z}}{\partial R}\right)$

$U_{z}=\frac{1}{R}\left(\frac{\partial\left(R \psi_{\emptyset}\right)}{\partial R}-\frac{\partial \psi_{r}}{\partial \emptyset}\right)$

To eliminate pressure terms in the momentum equations a curl of the momentum equations will be taken and the equations will be:

$$
\begin{aligned}
& R_{a}^{*} \frac{l}{\left(r_{2}-r_{1}\right)}\left(\frac{1}{R} \sin \delta \frac{\partial \theta}{\partial \emptyset}+\sin \emptyset \emptyset \cos \delta \frac{\partial \theta}{\partial Z}\right)=-\frac{\partial^{2} \psi_{r}}{\partial R^{2}}- \\
& \frac{1}{R^{2}} \frac{\partial\left(R \psi_{r}\right)}{\partial R}-\frac{2}{R} \frac{\partial \psi_{r}}{\partial R}-\frac{1}{R^{2}} \frac{\partial^{2} \psi_{r}}{\partial \emptyset^{2}}-\frac{\partial^{2} \psi_{r}}{\partial Z^{2}}-\frac{2}{R} \frac{\partial \psi_{z}}{\partial Z} \\
& R_{a}^{*} \frac{l}{\left(r_{2}-r_{1}\right)}\left(\cos \emptyset \cos \delta \frac{\partial \theta}{\partial Z}-\sin \delta \frac{\partial \theta}{\partial R}\right)=-\frac{\partial^{2} \psi_{\emptyset}}{\partial Z^{2}}-\frac{\partial^{2} \psi_{\emptyset}}{\partial R^{2}}- \\
& \frac{1}{R^{2}} \frac{\partial^{2} \psi_{\emptyset}}{\partial \emptyset^{2}}-\frac{2}{R^{2}} \frac{\partial \psi_{r}}{\partial \emptyset}+\frac{\psi_{\emptyset}}{R^{2}}-\frac{1}{R} \frac{\partial \psi_{\emptyset}}{\partial R}
\end{aligned}
$$


$R_{a}^{*} \frac{l}{\left(r_{2}-r_{1}\right)} \cos \delta\left(\frac{1}{R} \cos \emptyset \frac{\partial \theta}{\partial \emptyset}+\sin \emptyset \frac{\partial \theta}{\partial R}\right)=-\frac{\partial^{2} \psi_{z}}{\partial R^{2}}-\frac{1}{R} \frac{\partial \psi_{z}}{\partial R}-$

$\frac{1}{R^{2}} \frac{\partial^{2} \psi_{z}}{\partial \emptyset^{2}}-\frac{\partial^{2} \psi_{z}}{\partial Z^{2}}$

The energy equation is:

$\left(\frac{1}{R} \frac{\partial \psi_{z}}{\partial \emptyset}-\frac{\partial \psi_{\emptyset}}{\partial Z}\right) \frac{\partial \theta}{\partial R}+\frac{1}{R}\left(\frac{\partial \psi_{r}}{\partial Z}-\frac{\partial \psi_{z}}{\partial R}\right) \frac{\partial \theta}{\partial \emptyset}+\left(\frac{\psi_{\emptyset}}{R}+\frac{\partial \psi_{\emptyset}}{\partial R}-\right.$

$\left.\frac{1}{R} \frac{\partial \psi_{r}}{\partial \emptyset}\right) \frac{\partial \theta}{\partial Z}=\frac{l}{r_{1}}\left(\frac{\partial^{2} \theta}{\partial R^{2}}+\frac{1}{R} \frac{\partial \theta}{\partial R}+\frac{1}{R^{2}} \frac{\partial^{2} \theta}{\partial \emptyset^{2}}+\frac{\partial^{2} \theta}{\partial Z^{2}}\right)$

\subsection{Dimensionless Hydraulic Boundary Conditions}

The boundary conditions are given as:

$$
\begin{array}{ll}
\text { at } R=R_{1}, 1 & \frac{1}{R} \frac{\partial\left(R \psi_{r}\right)}{\partial R}=\psi_{\emptyset}=\psi_{z}=0 \\
\text { at } \emptyset=0, \pi & \psi_{r}=\frac{\partial \psi_{\emptyset}}{\partial \emptyset}=\psi_{z}=0 \\
\text { at } Z=0, L & \psi_{r}=\psi_{\emptyset}=\frac{\partial \psi_{z}}{\partial Z}=0
\end{array}
$$

\subsection{Dimensionless Thermal Boundary Conditions}

For the temperature field, the dimensionless thermal boundary conditions are:

$$
\begin{array}{ll}
\text { at } R=R_{1}=\frac{r_{1}}{r_{2}} & \theta=1 \\
\text { at } R=R_{2}=1 & \theta=0 \\
\text { at } \varnothing=0, \pi & \frac{\partial \theta}{\partial \emptyset}=0 \\
\text { at } Z=0, L & \frac{\partial \theta}{\partial Z}=0 \\
k_{\text {eff }}=(1-\varepsilon) k_{s}+\varepsilon k_{f}
\end{array}
$$

\section{COMPUTATIONAL TECHNIQUE}

The number of grid points used was 21 grid points in the $\mathrm{R}-$ direction, 31 in the $\phi$-direction and 301 in the $\mathrm{Z}$-direction which seems reasonable and will be used in the present study.

Convergence criterion given by inequality:

maximum error $=\left|\frac{\theta^{n+1}-\theta^{n}}{\theta^{n}}\right| \leq 10^{8}$

\subsection{Calculation of Local and Average Nusselt Number}

Local Nusselt number is the dimensionless parameter indicative of the rate of energy convection from a surface and can be obtained as follows [9]:

$N u=\frac{q\left(r_{2}-r_{1}\right)}{k\left(T_{1}-T_{2}\right)}$
The local Nusselt number $N u_{1}$ and $N u_{2}$ on the inner and the outer surfaces are written in the form [9]:

$$
\begin{aligned}
& N u_{\text {Local } 1}=-\left(1-R_{1}\right)\left(\frac{\partial \theta}{\partial R}\right)_{R=R_{1}} \\
& N u_{\text {Local } 2}=-\left(1-R_{1}\right)\left(\frac{\partial \theta}{\partial R}\right)_{R=R_{1}}
\end{aligned}
$$

The mean Nusselt number $N u_{i l}$ and $N u_{2}$ on the inner and the outer surfaces respectively are defined as:

$$
\begin{aligned}
& N u_{1}=-\left(1-R_{1}\right) \frac{1}{\pi L} \int_{0}^{\pi} \int_{0}^{L}\left(\frac{\partial \theta}{\partial R}\right)_{R=R_{1}} d Z d \emptyset \\
& N u_{2}=-\left(1-R_{1}\right) \frac{1}{\pi L} \int_{0}^{\pi} \int_{0}^{L}\left(\frac{\partial \theta}{\partial R}\right)_{R=1} d Z d \emptyset
\end{aligned}
$$

\section{RESULTS AND DISCUSSION}

\subsection{Isotherms and Streamlines Field}

The isothermal lines and streamlines within the toroidal ring are presented in a contour maps form in the $(\mathrm{R}-\phi)$ plane.

Fig. 2 shows the isothermal and streamlines respectively for a vertical toroidal loop $\delta=0, \mathrm{~L}=0.05, \boldsymbol{r}_{\mathbf{1}}=0.15 \mathrm{~m}$ and $\mathrm{Ra}=10$. The uniformity and symmetry of the isothermal lines is clear and it was observed that for $\mathrm{Ra}^{*}=5000$ in Fig. 3 the intensity of the streamlines is less than that for $\mathrm{Ra}=10$ and the cold region is larger because of the increase in heat transfer as $\mathrm{Ra}$ increase.
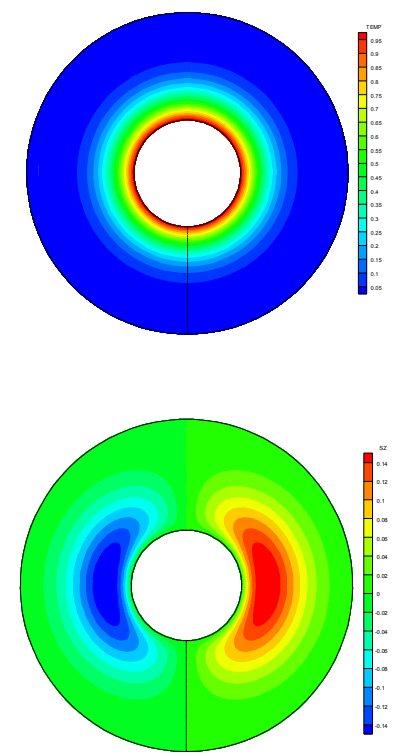

Fig. 2 Isothermal and streamlines contours for $\operatorname{Ra}^{*}=10$, $\delta=0$ and $r_{1}=0.15 \mathrm{~m}$ and $\mathrm{L}=0.05 \mathrm{~m}$ 

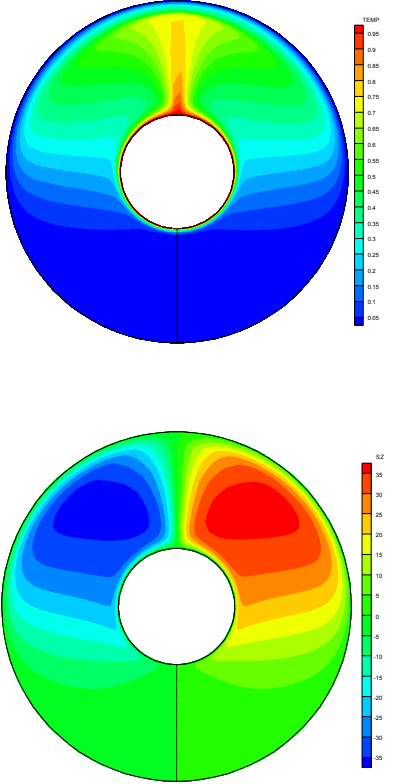

Fig. 3 Isothermal and streamlines contours for $L=0.05 \mathrm{~m}$, $\mathrm{Ra}^{*}=5000, \delta=0$ and $r_{1}=0.15 \mathrm{~m}$

Increase $\mathrm{Ra}^{*}$ and/or increase the inner radius results in a thicker cold layer near the bottom wall and a high temperature field near the top wall. More heat is transported upward, and a large difference of temperature is observed between the upper and lower parts of the toroidal loop as shown in Fig. 4
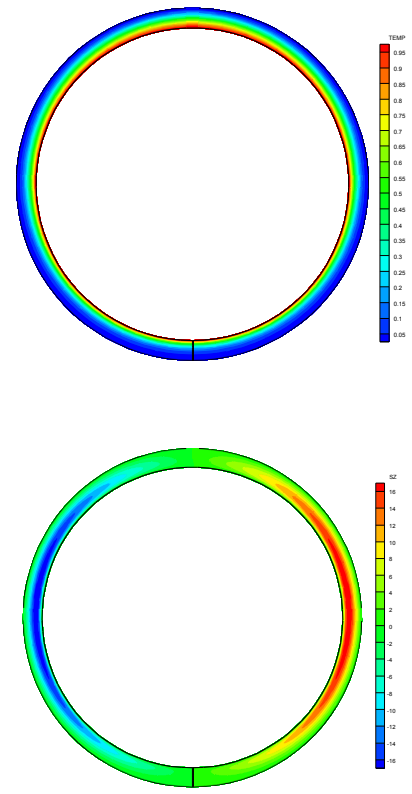

Fig. 4 Isothermal and streamlines contours for $\mathrm{Ra}^{*}=\mathbf{5 0 0 0}$ $\delta=0, \mathrm{~L}=0.05 \mathrm{~m}$ and $r_{1}=0.4 \mathrm{~m}$

Keeping the same parameters as in Fig. 4 but with $\delta=90$ (horizontal toroidal loop) in Fig. 5, the region seems very warm because the heat transfer is less in this situation so the temperature distribution is higher than that in Fig, 4.
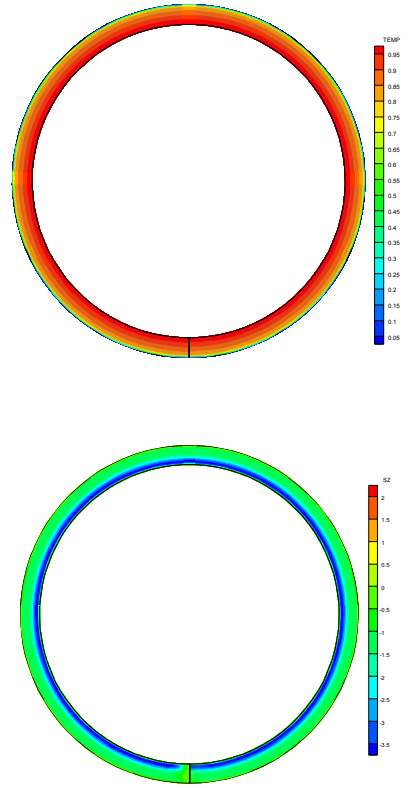

Fig. 5 Isothermal and streamlines contours for $\mathrm{Ra}^{*}=\mathbf{5 0 0 0}$ $\delta=90, \mathrm{~L}=0.05 \mathrm{~m}$ and $r_{1}=0.15 \mathrm{~m}$

Now increasing the length of the toroidal loop from 0.05 in Fig. 5 to 0.2 as in Fig. 6 cause to increase the heat transfer and the fluid will acquire more free movement due to the enlargement in the gap causing the temperature distribution to be less than that in Fig.5.
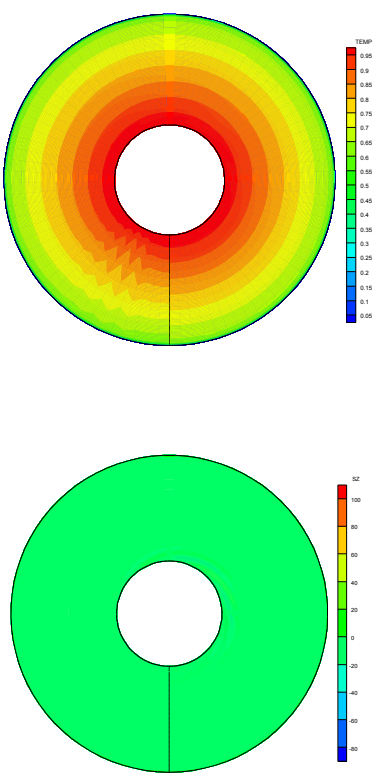

Fig. 6 Isothermal and streamlines contours for $\mathrm{Ra}^{*}=5000$ $\delta=90, \mathrm{~L}=0.2 \mathrm{~m}$ and $r_{1}=0.15 \mathrm{~m}$

The local Nusselt number in the angular direction on the cold outer surface and the inner hot surface of the toroidal loop are illustrated for $\mathrm{Ra}=10$ and $\mathrm{Ra}=5000$ in Fig. 7 and Fig. 8 respectively for the same other parameters. Fig. 7 shows a smooth curve with lesser values than that in Fig. 8 due to the increase in $\mathrm{Ra}$ which causes an increase in heat transfer and 
consequent the local Nusselt number which is an indication of heat transfer will be increased.
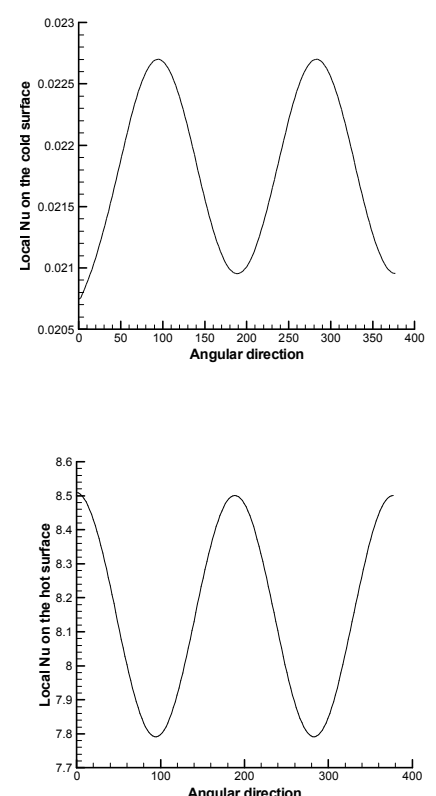

Fig. 7 Local Nusselt number in the angular direction for $\mathrm{Ra}^{*}=10, \delta=0, \mathrm{~L}=0.05 \mathrm{~m}$ and $r_{1}=0.15$
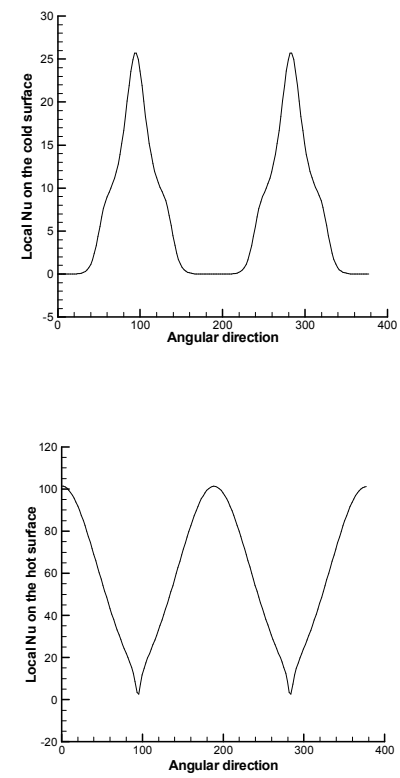

Fig. 8 Local Nusselt number in the angular direction for $\mathrm{Ra}^{*}=50000, \delta=0, \mathrm{~L}=0.05 \mathrm{~m}$ and $r_{1}=0.15 \mathrm{~m}$

Fig. 9 shows the increase of the mean Nusselt number with Ra number for vertical toroidal loop $(\delta=0)$ and for a small gap of length $\mathrm{L}=0.05$ with $r_{1}=0.15$ on the cold outer surface and the inner hot surface. It is clear that for low values of $\mathrm{Ra}$ the increase in the mean $\mathrm{Nu}$ is small and as $\mathrm{Ra}$ exceeds 500 the increase in $\mathrm{Nu}$ is very significant.

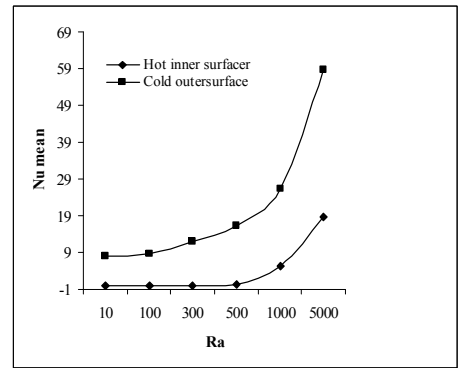

Fig. 9 The variation of the mean Nusselt number with Ra number for $\delta=0 \mathrm{~L}=0.05$ and $r_{1}=0.15$

Fig. 10 shows the variation of the mean Nusselt number with the radius ratio $\left(r_{1} / r_{2}\right)$ on the cold surface for two dimensionless gap lengths 0.05 and 0.2 and it is clear that for small values of radius ratio which means large toroidal gap the mean Nusselt number is very high and when $\left(r_{1} / r_{2}\right)$ increase up 0.555 the two curves will be coincident and its value will be decreased significantly.

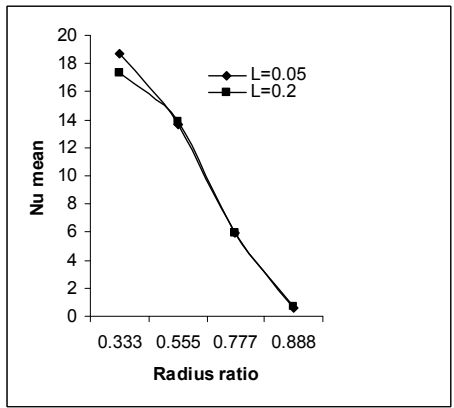

Fig. 10 The variation of the mean Nusselt number on the cold surface with the radius ratio $\left(r_{1} / r_{2}\right)$ for $R a=5000, \delta=0$

Fig. 11 shows the variation of the mean Nusselt number with the radius ratio $\left(\mathrm{r}_{1} / \mathrm{r}_{2}\right)$ on the hot inner surface for two dimensionless gap lengths 0.05 and 0.2 and it is clear that for small values of radius ratio which means large toroidal gap the mean Nusselt number is very high and when $\left(r_{1} / r_{2}\right)$ increase the mean $\mathrm{Nu}$ decrease and the two curves will be coincident at about $\left(\mathrm{r}_{1} / \mathrm{r}_{2}\right)$ equal 0.777 .

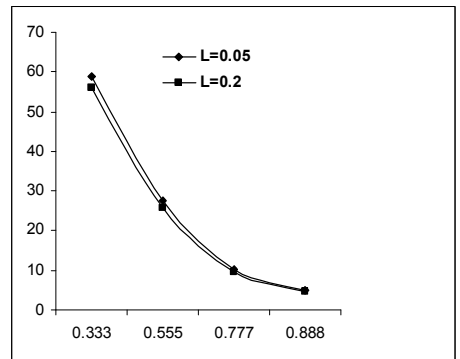

Fig. 11 The variation of the mean Nusselt number on the hot surface with the radius ratio $\left(r_{1} / r_{2}\right)$ for $R a=5000, \delta=0$

Fig. 12 shows that the mean $\mathrm{Nu}$ is nearly constant for low values of $\mathrm{Ra}$ and remains constant for small toroidal gap, but as $r_{1}$ decrease the mean $\mathrm{Nu}$ increase significantly. It seems that $r_{1}=0.15 \mathrm{~m}$ is the best radius which gives the highest heat transfer. 


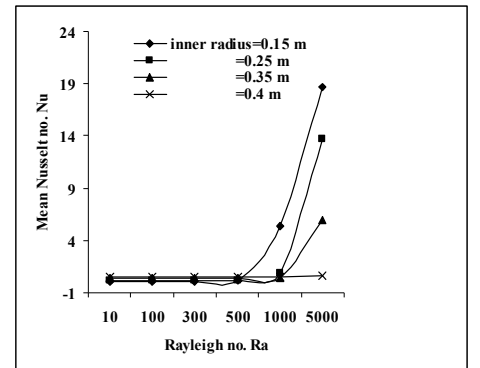

Fig. 12 The variation of the mean Nusselt number on the outer cold surface with $\operatorname{Ra}$ for $\delta=0$ and various values of the inner radius

Fig. 13 and Fig. 14 show the variation of the mean $\mathrm{Nu}$ on the cold outer surface of the toroidal loop for vertical and horizontal toroidal loop respectively. The two figures show that the curves coincide with each other for lowest and highest values of $\mathrm{Ra}$. In the ranges of Ra between about 300 to 800 the lowest heat transfer is observed for $\mathrm{L}=0.05$ at $\mathrm{Ra}=500$, so it appears to be the best design of such devices to take $\mathrm{L}=0.05$ with inner radius of $0.15 \mathrm{~m}$ and to use it at $\delta=0$ (vertical) which gives low heat transfer that mean the device keep the heat for a long time with high temperature distribution.

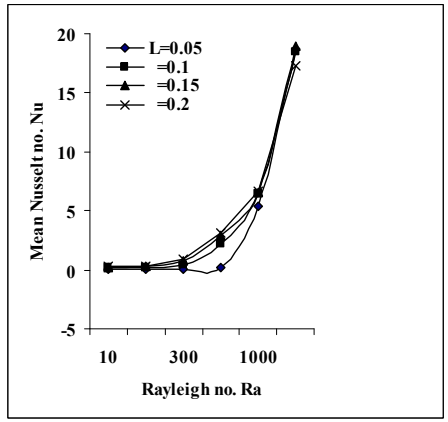

Fig. 13 The variation of the mean Nusselt number on the outer cold surface with $R a$ for $\delta=0$ and various values of the gap length

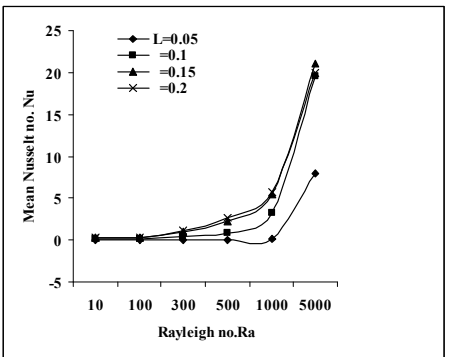

Fig. 14 The variation of the mean Nusselt number on the outer cold surface with $\operatorname{Ra}$ for $\delta=90$ and various values of the gap length

\section{CONCLUSIONS}

The following major conclusions can be drawn from the study:

1- The vertical toroidal loop is more suitable than the horizontal one because the heat transfer is less so the temperature values will be higher and remain high for a longer time which is required in such devices.
2- The best design of such devices is to take $\mathrm{L}=0.05$ with inner radius of $0.15 \mathrm{~m}$ and to use it at $\delta=0$ (vertical)

3- For future it is recommended to study the toroidal loop using composite material with low thermal conductivity to decrease the heat transfer and it is recommended to repeat this research using nanofluid as a working fluid.

\section{REFERENCES}

[1] G.D. Raithby, K.G.T. Hollands, Natural convection, in: W.M. Rohsenow et al. (Eds.), Handbook of Heat Transfer, third ed., McGraw-Hill, New York, 1998 (Chapter 4).

[2] H. Manal saleh, Enhancement of Heat Transfer in Horizontal Annulus Filled with Porous Media under Constant and Periodic Boundary Conditions, Journal of Mechanical Engineering. Photon 124 (2014) 198-209.

[3] Dalia Sabina Cimpean, Ioan Pop, Fully developed mixed convection flow of a nanofluid through an inclined channel filled with a porous medium, International Journal of Heat and Mass Transfer 55 (2012) 907-914.

[4] H. Saleh, R. Roslan, I. Hashim, Natural convection heat transfer in a nanofluid-filled trapezoidal enclosure, International Journal of Heat and Mass Transfer 54 (2011) 194-201.

[5] El Hassan Ridouane, Christopher M. Danforth, Darren L. Hitt, A 2-D numerical study of chaotic flow in a natural convection loop, International Journal of Heat and Mass Transfer 53 (2010) 76-84.

[6] Carlo Alberto Tiberio, Luigi Raganella, Germana Banci, Cafiero Franconi, The RF Toroidal Transformer as a Heat Delivery System for Regional and Focused Hyperthermia, IEEE Transactionson Biomedical Engineering. Vol. 35, No. 12, December 1988.

[7] G. Refai-Ahmed, M. M. Yovanovich, D. Cooper, Heat Transfer Modelling of Toroidal Inductors: Effect of Orientations, IEEE Intersociety Conference on Thermal Phenomena 1998.

[8] Nield D. A., Bejan A., 1999. Convection in Porous Media, Springer-Verlag, New York.

[9] Fukuda K., Takata Y., Hasegawa S., Shimomura H. and Sanokawa K., 1980, Three - Dimensional Natural Convection in a Porous Medium Between Concentric Inclined Surfaces, Proc. $19^{\text {th }}$ Natl Heat Transfer Conf., Vol. HTD -8, $97-103$.

[10] Wang $\mathrm{Bu}$ - Xuan and Zhang Xing, 1990, Natural Convection in Liquid Saturated Porous Media Between Concentric Inclined Surfaces, Int. J. Heat and Mass Transfer Vol. 33. No 5, 827-833.

[11] Aziz K. and Hellums J. D., 1967, Numerical Solution of the Three Dimensional Equations of Motion for Laminar Natural Convection, The Physics of Fluids, Vol. 10, No. $2,314-324$.

[12] H. Manal AL- Hafidh, A. Khawla AL Zubaidy, Heat Transfer Investigation of $\mathrm{TiO} 2$ Nanofluid in Silica Aerogel/Glass Fiber Composite Material Enclosure, International Journal of Computer Applications, Vol. 112- No. 8, February 2015. 